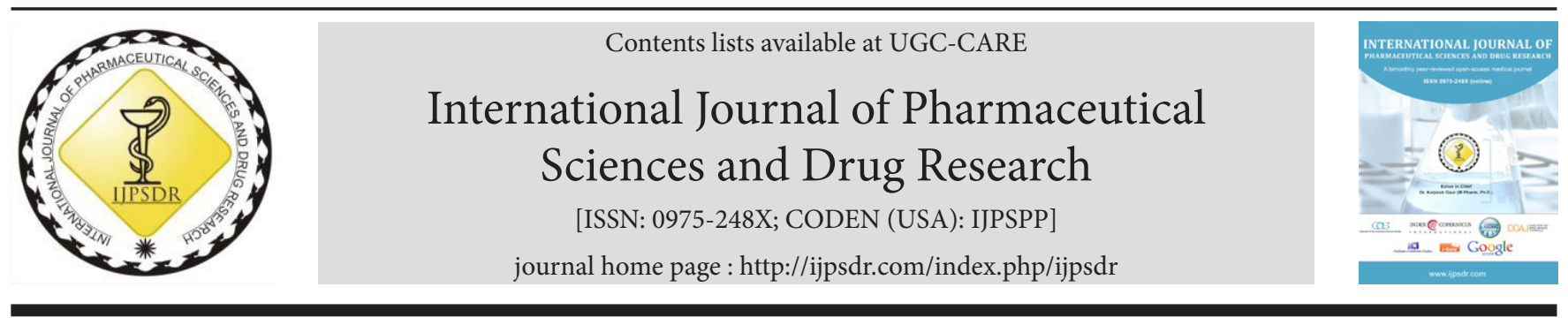

Research Article

\title{
Computational Studies of Vitexin isolated from Vitex negundo against Rheumatoid Arthritis Targets
}

\section{R. Gayathri Devi*, S. Bhagavathy}

PG and Research Department of Biochemistry, Mohamed Sathak College of Arts and Science (University of Madras), Sholinganallur, Chennai-600119, Tamil Nadu, India

\begin{tabular}{l} 
A R T I C L E I N F O \\
\hline Article history: \\
Received: 05 May, 2020 \\
Revised: 23 August, 2020 \\
Accepted: 30 August, 2020 \\
Published: 30 September, 2020 \\
Keywords: \\
AutoDock Vina, \\
BIOVIA discovery studio \\
visualizer, \\
Human inhibitory kinase $\beta$, \\
Molecular docking, \\
Rheumatoid arthritis, \\
TNF $\alpha$ converting enzyme, \\
TNF $\alpha$ receptor 1, Vitexin. \\
DOI: \\
10.25004/IJPSDR.2020.120510
\end{tabular}

\begin{abstract}
A B S T R A C T
One chronic inflammatory autoimmune disease that needs attention to be treated is rheumatoid arthritis (RA). Especially, RA encounters more female cases that manifest progressive destruction of bone and cartilage. Expression of more inflammatory cytokines and matrix metalloproteins at the synovial tissue destruct collagen structure and worsen the condition. Generally, anti-inflammatory drugs and immunemodulatory agents are used to deduce the progression of the disease. Such chemical entities have proven many side effects and expensive. This study provides a glimpse of structural based drug designing, using bioactive pharmakon, from a folk chloric herb. Vitex negundo is a traditionally available plant that has been reported to possess pharmaco-beneficiary bioactive flavonoids, especially vitexin. Virtual screening involves docking of vitexin with three different targets responsible for triggering the collagenase enzyme achieved using AutoDock. The binding energy of vitexin and TNF $\alpha$ receptor1 (TNFR1) was found to be slightly higher when compared to docking results of TNF $\alpha$ converting enzyme (TACE) and human inhibitory kinase $\beta$ (hIK $\beta$ ) proteins. The molecular interactions were visualized using BIOVIA discovery studio visualizer. This study may become evident that vitexin has enhanced pharmacological benefits that need to be ensured through in vitro and in vivo assays.
\end{abstract}

\section{INTRODUCTION}

Rheumatoid arthritis (RA) is a progressive chronic inflammatory autoimmune disease that leads to the destruction of cartilage and bone. More than $15 \%$ of the world's populations are affected by encountering higher female cases. Since the exact cause is not known, disease-modifying anti-inflammatory drugs (DMARDs) and non-steroidal anti-inflammatory drugs (NSAIDs) are administered even in the advanced stages of RA. ${ }^{[1]}$ DMARDs and NSAIDs are better immuno-modulatory agents reported adverse side effects after prolonged use. ${ }^{[2]}$ Natural molecular entities may replace chemical leads in healing diseases. Biological compounds are cost-effective, ease of production, safety, and efficacy levels also high..$^{[3]}$ $V$. negundo is an annual herb available throughout India, commonly called as "nirgundi" by Ayurvedic pharmacopoeia of India reported excellent anti-inflammatory property, which may occur due to the presence of bioactive pharmakon, vitexin. ${ }^{[4]}$ As an alternate, a biological pharmakon isolated from $V$. negundo may satisfy the above need. Vitexin is a flavone glycoside considered an important flavonoid of interest that possesses ample pharmacological benefits. ${ }^{[5]}$ It is reported that vitexin serves as an excellent anti-inflammatory pharmakon, which reduces the level of cytokine expression at the site of inflammation. ${ }^{[6]}$ TNF $\alpha$ is an important cytokine that plays a crucial role in the immune system's signaling

\footnotetext{
${ }^{*}$ Corresponding Author: R. Gayathri Devi

Address: Research Scholar, PG and Research Department of Biochemistry, Mohamed Sathak College of Arts and Science, Sholinganallur, Chennai-600119, Tamil Nadu, India

Email $\bowtie$ : gaya3dev20msc@gmail.com

Tel.: +91-8778583620

Relevant conflicts of interest/financial disclosures: The authors declare that the research was conducted in the absence of any commercial or financial relationships that could be construed as a potential conflict of interest.

Copyright (C 2020 R. Gayathri Devi et al. This is an open access article distributed under the terms of the Creative Commons Attribution- NonCommercialShareAlike 4.0 International License which allows others to remix, tweak, and build upon the work non-commercially, as long as the author is credited and the new creations are licensed under the identical terms.
} 
pathways and inflammation. ${ }^{[7]}$ TACE is responsible for the activation of TNF $\alpha$ to bind its receptor TNFR. ${ }^{[8]} \mathrm{A}$ human inhibitory kinase $(\operatorname{Ik} \beta)$ upon activation facilitates the upregulation of translocated NFk $\beta$ at the nuclear level and enhances matrix-metallo protein expression. ${ }^{[9]}$ Matrix metallo proteins (MMPs) abundant in synovial tissue, once activated may significantly destruct extracellular matrix, and erode bone and cartilage. ${ }^{[10]}$ Attenuation of TACE, TNFR1, and human IK $\beta$ may reduce the sequestering of more inflammatory cytokines and MMPs.

Structure-based drug discovery promotes a better understanding of three-dimensional protein targets that actively performs in a disease. Computer-aided drug discovery may highlight and scientifically validate the traditional folkloric applications of certain herbs and their biologically active compounds. ${ }^{[1]}$ The efficacy of vitexin to repress the above targets may examine by computational molecular docking analysis. The pharmacokinetic profile of the vitexin has been evaluated to check the pharmacological potency. Virtual screening may help to assure drug-likeness of vitexin and may pay the way for further in vitro and in vivo studies in the treatment of rheumatoid arthritis.

\section{Materials AND Methods}

\section{Protein Preparation}

The three-dimensional structure file of drug targets, such as, TACE, TNFR1, and hIK $\beta$ was retrieved from the Research Collaboratory for Structural Bioinformatics-Protein data Bank (RCSB-PDB) database. All water molecules were removed, and Gasteiger charges were assigned to the protein structure. ${ }^{[12]}$

\section{Ligand Preparation}

SMILES structure of vitexin retrieved from PubChem was submitted in SwissADME, an online tool that predicts the selected compound's efficiency by checking various physicochemical properties. ${ }^{[13]}$ In silico toxicity predictor tool predicts the lethal dose $50\left(\mathrm{LD}_{50}\right)$ value in $\mathrm{mg} / \mathrm{kg}$ body weight. Protox II, an online tool, predicts and assesses the toxicity of vitexin (Table 1). ${ }^{[14]}$ 2D structure of vitexin was drawn using ChemSketch from the SMILES data available in the PubChem database. The 3D structure of vitexin was generated, optimized, and saved in a mol file. Further conversions were made using the open babel molecular converter program and saved in PDB format. ${ }^{[12]}$

\section{Protein-Ligand Interaction Prediction}

AutoDock is one of the best suites of automated docking tools. The software is used for modeling flexible small molecules, such as, drug molecule binding to target proteins of known structure. Genetic algorithms are used in this suite for checking the conformational search. AutoDock is a user-friendly tool to perform blind docking, where the location of the binding site is not known. Molecular docking was performed using the AutoDock Tools 4.2 graphical user interface, which generates ten conformations (poses) of the proteinligand complex customized in the order from lowest to highest binding free energy $(\Delta G) \cdot{ }^{[15]}$

\section{BIOVIA Discovery Studio Visualizer}

BIOVIA visualization tool helps to visualize the docking interactions between vitexin and different protein targets of interest in both 2D and 3D. Emphasized images of different interactions are easily visualized in the Discovery Studio tool. ${ }^{[16]}$

\section{RESUlt AND Discussion}

\section{Vitexin Structure and its Properties}

The 2D (Fig. 1) and 3D structure (Fig. 2) of vitexin were retrieved and saved in PDB format. Membrane permeability and bioavailability of vitexin are always assessed with some basic molecular properties, such as, log $\mathrm{p}$ (partition coefficient), molecular weight (MW), or counts of hydrogen bond acceptors and donors in a molecule. ${ }^{[17]}$ These molecular properties were used in formulating the "rule of five."[18] The rule states that most molecules with good membrane permeability have molecular weight $\leq 500$, calculated octanol-water partition coefficient, log $\mathrm{P} \leq 5$, hydrogen bond donors $\leq 5$, and acceptors $\leq 10$. ${ }^{[19]}$

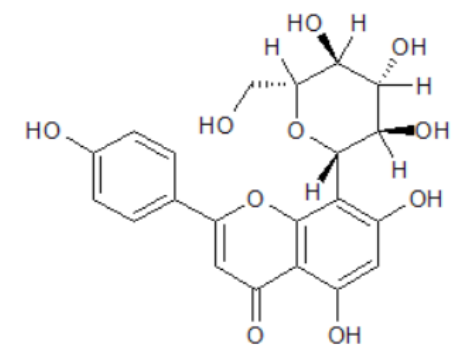

Fig. 1: 2D structure of vitexin

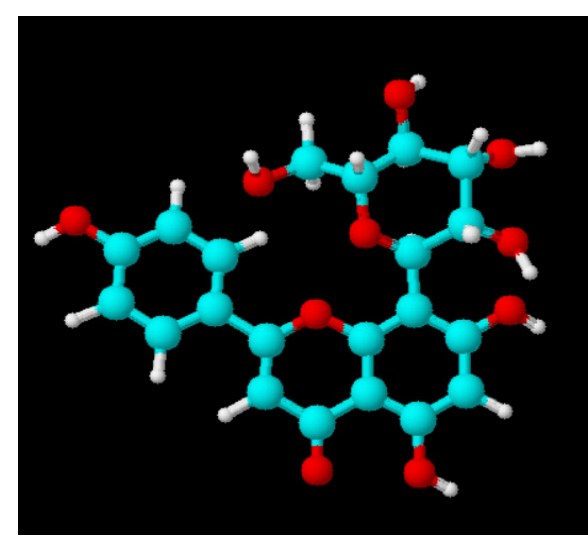

Fig. 2: 3D structure of vitexin

Table 1: $\mathrm{LD}_{50}$ and toxicity class for vitexin

\begin{tabular}{lll}
\hline Ligand name & $L D_{50} \mathrm{mg} / \mathrm{kg}$ & Toxicity class \\
\hline Vitexin & 1,213 & 4 \\
\hline
\end{tabular}


Therefore, Lipinski's rule of five was used to test the bioavailability characteristics, such as, absorption, distribution, metabolism, elimination (ADME) of the lead compounds, which was predicted by molinspiration ${ }^{[20]}$ and SwissADME.

Physicochemical properties depicting oral bioavailability was predicted using SwissADME (Table 2), in which lipophilicity was calculated in terms of XLOGP3 as 0.21 . Insolubility log value depicted as -2.84 indicates solubility of vitexin, and unsaturation as per Csp3 was found to be 0.29 (Fig. 3). ${ }^{[21]}$ Properties, such as, G-protein coupled receptor (GPCR) ligand, ion channel modulator, kinase inhibitor, nuclear receptor ligand, protease inhibitor, and enzyme inhibitor, were predicted with Molinspiration, and bioactive scores were tabulated (Table 3). ${ }^{[22]}$

\section{Molecular Docking of Vitexin against Drug Targets}

AutoDock 4.2 suite was used to perform molecular docking analysis of vitexin against TACE, TNFR1, and hIK $\beta$ b. Hydrogen atoms were added, water molecules removed, and Gasteiger charges were added to the ligand and protein. The rotatable bonds of the ligand were altered using the "choose torsions" option. Further flexible bonds of the ligand were checked to dock with rigid protein targets. Blind docking was performed using grid point value $(\mathrm{X}, \mathrm{Y}$, and $\mathrm{Z})$ of $126 \AA$ and spacing between the grid points was $0.375 \AA$. The Lamarckian genetic algorithm was selected for ligand conformational searching, ${ }^{[23]}$ and default docking parameters were used. A total of 10

Table 2: SWISSADME-predicted Lipinski rule values, including TPSA, bioavailability, and water solubility for vitexin

\begin{tabular}{ll}
\hline Properties & Predicted value \\
\hline Mi Logp & 0.52 \\
TPSA & 181.05 \\
Number of atoms & 31 \\
Mw & 432.38 \\
H bond acceptor & 10 \\
H bond donor & 7 \\
Number of violations & 1 \\
Number of rotational bonds & 3 \\
Volume & 355.2 \\
Bioavailability & 0.55 \\
Water solubility & Soluble \\
\hline & Table 3: Bioactivity score for vitexin \\
\hline Bioactivity & Probability score \\
\hline GPCR ligand & 0.13 \\
Ion channel modulator & 0.14 \\
Kinase inhibitor & 0.19 \\
Nuclear receptor ligand & 0.23 \\
Protease inhibitor & 0.03 \\
Enzyme inhibitor & 0.46 \\
\hline
\end{tabular}

docking configurations were determined in each docking calculation.

A preferable docking configuration was chosen based on the lowest empirical binding free energy and the most frequent cluster. AutoDock results are ranked according to the highest negative binding free energies and corresponding root mean square deviation (RMSD) values from the experimentally determined binding site. AutoDock shares functional commonalities, including the global optimization of the scoring function, precalculation of grid maps, and the pre-calculation of distant dependent pair-wise energetics between each atom type. However, they employ a different scoring function and algorithms to obtain binding free energies and should be considered as different programs. ${ }^{[24]}$ The docked protein-ligand complex (Table 4) visualized using BIOVIA Discovery Studio visualizer, and the distances of interactions are calculated (Table 5).

\section{Visualization of Protein-Vitexin Complex Interactions}

The best-docked ligand models were selected according to the lowest binding energy. Two and three-dimensional conformational structures of the ligand-protein complexes were visualized using BIOVIA Discovery Studio Visualizer v.4. $5^{[25]}$ to investigate the binding modes. The ligand binds at the target protein by non-covalent interactions, such as, H-bonding, alkyl, alkyl- $\pi, \pi-\pi, \pi-\sigma$, and van der Waals interactions. Simplified visualization is illustrated in 2D, which displays the H-bonding, van der Waals forces, carbonoxygen dipole-dipole interaction, alkyl-pi interactions, T-shaped pi-pi stacking, and pi-pi stacking. Images

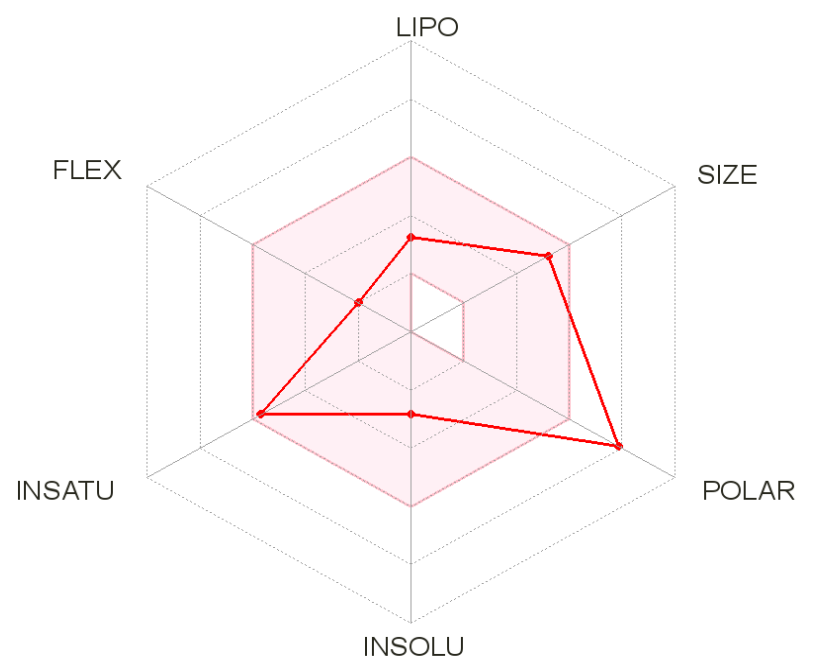

Fig. 3: Bioavailability radar (pink zone depicts suitable physicochemical space for oral bioavailability) for vitexin (LIPO indicates lipophilicity in terms of XLOGP3, SIZE indicates in terms of molecular weight, POLAR indicates polarity in terms of topological polar surface area, INSOLU depicts insolubility in water in terms of $\log \mathrm{S}$ scale, INSATU refers to unsaturation as per fraction of carbons in the sp3 hybridization, and finally FLEX indicates flexibility as per rotatable bonds) 
R. Gayathri Devi et al.

Table 4: Docking score predictions of protein-vitexin complex

\begin{tabular}{lllll}
\hline S. No. & Conformation parameters & TACE & TNFR1 & hIKRb \\
\hline 1 & Binding energy & -5.71 & -6.1 & -4.7 \\
2 & Inhibitory constant $(\mu \mathrm{m})$ & 65.22 & 33.94 & 360.35 \\
3 & van der Waal interaction & -8.39 & -8.64 & -7.32 \\
4 & Electrostatic energy & -0.3 & -0.44 & -0.36 \\
5 & Torsional energy & 2.98 & 2.98 & 2.98 \\
6 & Unbound energy & -7 & -6.66 & -7.54 \\
7 & Amino acids & SER287, LEU285, TYR238, & CYS158, CYS127, GLN131, GLN548, ARG549, ARG452, & GLN548, GLU149, CYS99 \\
\hline
\end{tabular}

Table 5: Predicted distance and interacted amino acid residues of protein targets with vitexin

\begin{tabular}{|c|c|c|c|}
\hline Vitexin (ligand) & AA-residue & Distance $(\AA))$ & $\begin{array}{l}\text { Docking } \\
\text { energy }(\mathrm{kcal} / \mathrm{mol})\end{array}$ \\
\hline \multirow[t]{6}{*}{ TACE (protein) } & SER287 & 3.36 & \multirow[t]{6}{*}{-5.71} \\
\hline & LEU285 & 2.94 & \\
\hline & TYR238 & 2.186 & \\
\hline & GLU463 & 2.086 & \\
\hline & PR0218 & 2.149 & \\
\hline & PRO218 & 1.983 & \\
\hline \multirow[t]{6}{*}{ TNFR1 (protein) } & CYS158 & 2.07 & \multirow[t]{6}{*}{-6.1} \\
\hline & CYS127 & 2.017 & \\
\hline & CYS127 & 2.48 & \\
\hline & CYS127 & 1.82 & \\
\hline & GLN131 & 1.822 & \\
\hline & ASN130 & 3.3 & \\
\hline \multirow[t]{7}{*}{ hIK $\beta b$ (protein) } & GLU149 & 2.71 & \multirow[t]{7}{*}{-4.7} \\
\hline & GLU97 & 2.96 & \\
\hline & CYS99 & 2.93 & \\
\hline & GLN548 & 1.956 & \\
\hline & ARG549 & 2.042 & \\
\hline & ARG452 & 1.817 & \\
\hline & GLN548 & 2.199 & \\
\hline
\end{tabular}

were generated using Discovery Studio Visualizer 4.5. ${ }^{[26]}$ Interactions between TNFR1 and vitexin (Fig. 4) showed the lowest binding energy when compared to TACE-vitexin interactions (Fig. 5) and vitexin-hIK $\beta$ b kinase protein binding interactions (Fig. 6).

Computational analysis is less laborious, easy to perform, and yield quick results than conventional drug designing techniques. Cost-effective computational approaches may also seem to screen docking hits to produce small novel herbal-based drugs. AutoDock Vina docking enrolls better-performing speed simultaneously, elucidate better result accuracy. ${ }^{[27]}$ The resulting docking complexes are expected to produce an excellent pharmacological effect in the treatment of RA. This investigation may serve as a tool for the evaluation of drug-like properties of pharmakon from folkloric herb

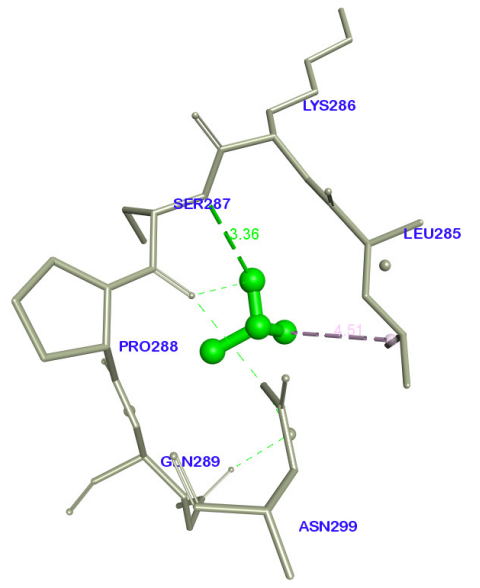

a) 3D interactions

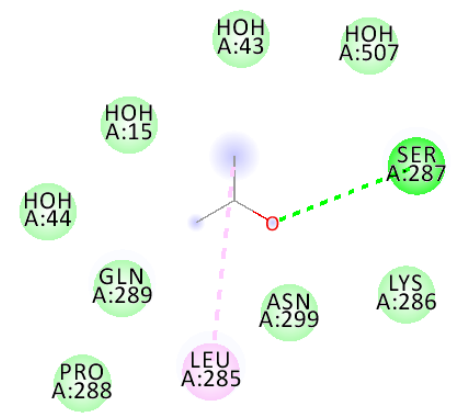

Interactions
$\square$ van der Waals
Conventional Hydrogen Bond

\section{$\square$ Alky \\ b) $2 \mathrm{D}$ interactions}

Fig. 4: 3D and 2D representations of vitexin-TACE protein interactions visualized by BIOVIA Discovery Studio visualizer; a) 3D interactions: TACE (protein)-line model; vitexin (ligand)-ball and stick model; green dotted lines-hydrogen bond interactions; b) 2D diagram: Ligand line model and protein interactions are colored depending on their type: conventional hydrogen bonds are colored in green, van der Waals interactions are colored in light green, and $\pi$-alkyl interactions are colored in light pink, respectively; the blue halo surrounding the interacting residues represents solvent-accessible surface that is proportional to its diameter 


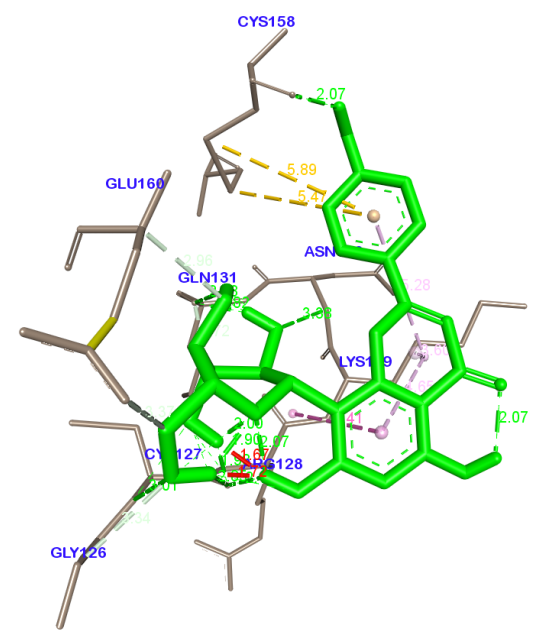

a) 3D interactions
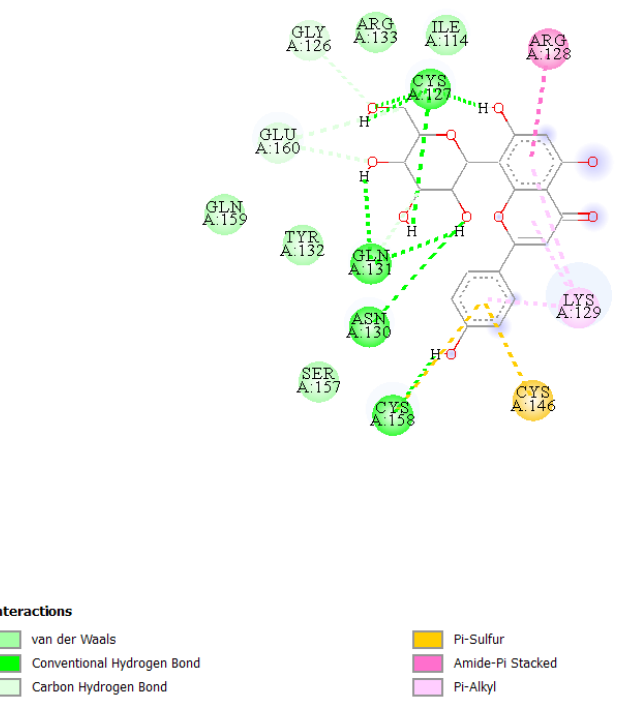

Carbon Hydrogen Bon

\section{b) $2 \mathrm{D}$ interactions}

Fig. 5: 3D and 2D representations of vitexin-TNRF1 receptor protein interactions visualized by BIOVIA Discovery Studio visualizer;

a) 3D interactions: TNFR1 (protein)-line model; vitexin (ligand)-ball and stick model; green dotted lines-hydrogen bond interactions;

b) 2D diagram: Ligand line model and Protein interactions are colored depending on their type; conventional hydrogen bonds are colored in green, van der Waals interactions are colored in light green, $\pi$-sulfur interactions are colored in yellow, amide- $\pi$ stacked, and $\pi$-alkyl interactions are colored dark pink and light pink, respectively; the blue halo surrounding the interacting residues represents the solventaccessible surface that is proportional to its diameter

$V$. negundo. It can be further utilized for the beneficiary of mankind after in vivo and clinical trial studies.

\section{CONCLUSION}

The present computational studies, concludes that vitexin might serve as better potent herbal pharmakon in repressing the activated inflammatory protein targets of RA such as, TACE, TNFR1, and hIK $\beta$ b. Vitexin from $V$. negundo might serve as an efficient pharmaco-beneficiary

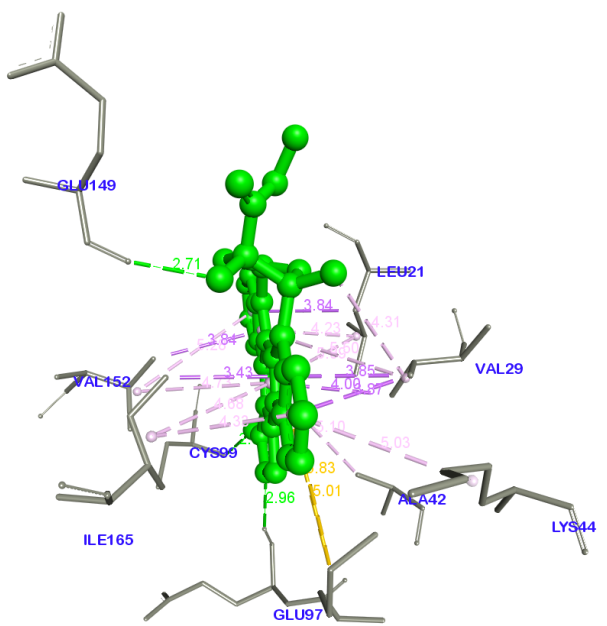

a) 3D interactions
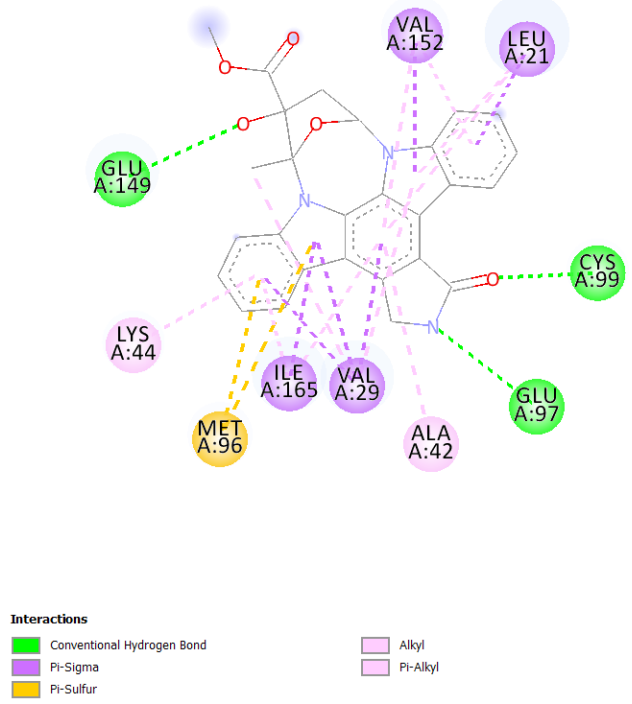

Allkyl
Pi-Alkyl

b) $2 \mathrm{D}$ interactions

Fig. 6: 3D and 2D representations of vitexin- hIK $\beta$ b kinase protein interactions visualized by BIOVIA Discovery Studio visualizer; a) 3D interactions: hIK $\beta$ b (protein)-line model; vitexin (ligand)-ball and stick model; green dotted lines-hydrogen bond interactions;

b) 2D diagram: Ligand line model and protein interactions are colored depending on their type; conventional hydrogen bonds are colored in green, $\pi$-sulfur interactions are colored in yellow, $\pi$-sigma interactions are colored purple, alkyl and $\pi$-alkyl interactions are colored light pink, respectively; the blue halo surrounding the interacting residues represents the solvent-accessible surface that is proportional to its diameter

lead in replacement of commercial drugs due to its lesser side effects and cost effectiveness, thereby the folk-chloric bioactive compound vitexin might act as a promising lead in therapeutics of Rheumatoid arthritis.

\section{REFERENCES}

1. Verma MK, Sobha K. Understanding the major risk factors in the beginning and the progression of rheumatoid arthritis: current scenario and future prospects. J Inflamm Res. 2015;64:647-659.

2. Benjamin O, Bansal P, Goyal A, Lappin SL. Disease Modifying AntiRheumatic Drugs (DMARD). Star Pearls Publishing. 2020. 


\section{R. Gayathri Devi et al.}

3. Veeresham C. Natural products derived from plants as a source of drugs. J Adv Pharm Technol Res. 2012;3:200-201

4. Roy, Somendu. Analysis of flavonoids and iridoids in Vitex negundo Linn by HPLC-PDA and method validation. Natural product communications. 2013.

5. Yahaya M, Zolkiffly S, Moklas M, Abdul Hamid H,Stanslas J ,Zainol $\mathrm{M}$, et al. Possible Epigenetic Role of Vitexin in Regulating Neuro inflammation in Alzheimer's Disease. J Immunology Research. 2020;7.

6. Nikfarjam BA, Hajiali F, Adineh M, Nassiri-Asl M. Anti-inflammatory Effects of Quercetin and Vitexin on Activated Human Peripheral Blood Neutrophils:-The effects of quercetin and vitexin on human neutrophils. J Pharmacopuncture. 2017;20(2):127-131.

7. Awaluddin R, Muhtadi WK, Chabib L, Ikawati Z, Martien R, Ismail H. Molecular docking and ADME-toxicity studies of potential compounds of medicinal plants grown in Indonesia as an antirheumatoid arthritis. InAIP Conference Proceedings 2017 Mar 17 (Vol. 1823, No. 1, p. 020033). AIP Publishing LLC.

8. Wong E, Cohen T, Romi E, Levin M, Peleg Y, Arad U, Yaron A, Milla ME, Sagi I. Harnessing the natural inhibitory domain to control TNF $\alpha$ converting enzyme (TACE) activity in vivo. Scientific reports. 2016 Dec 16;6:35598.

9. Matthew P, Vincenti, constance E Brinckerhoff. Transcriptional regulation of collagenase (MMP-1, mmP-13) genes in arthritis: integration of complex signalling pathways for the recruitment of gene-specific transcription factors. http://arthritis-research. com/content/4/3/157. 2001

10. Zhang LM, Zhou JJ, Luo CL. CYLD suppression enhances the proinflammatory effects and hyperproliferation of rheumatoid arthritis fibroblast-like synoviocytes by enhancing NF- $\mathrm{B}$ activation. Arthritis Res Ther. 2018; 20(1):219.

11. Lionta E, Spyrou G, Vassilatis DK, Cournia Z. Structure-based virtual screening for drug discovery: principles, applications and recent advances. Curr Top Med Chem. 2014;14(16):1923-1938.

12. Syed Mohd, Shazi Shakil, Mohd. Haneef. A simple click by click protocol to perform docking:Autodock 4.2 made easy for nonbioinformaticians. EXCLI Journal. 2013

13. Daina A, Michielin O, Zoete V. SwissADME: A free web tool to evaluate pharmacokinetics, drug-likeness and medicinal chemistry friendliness of small molecules. Sci Rep. 2017;7:42717.

14. Banerjee P, Eckert AO, Schrey AK, Preissner R. ProTox-II: A webserver for the prediction of toxicity of chemicals. Nucleic Acids Res. 2018;46:W257-263

15. Shah N, Rao H, Verma SK. Structure-based drug designing methodology to elucidate the binding affinities of phyto-ligands of Annona muricata against oncogenic PirH2. MOJ Proteomics Bioinform. 2018;7(2):113-120.

16. Dhananjayan K. Molecular docking study characterization of rare flavonoids at the Nac-binding site of the first bromodomain of BRD4 (BRD4 BD1). Journal of Cancer Research. 2015;2015.

17. Ertl P, Rohde B, Selzer P. Fast calculation of molecular polar surface area as a sum of fragment-based contributions and its application to the prediction of drug transport properties. J Med Chem. 2000; 43 (20):3714-3717.

18. Lipinski CA, Lombardo F, Dominy BW, Feeney PJ. Experimental and computational approaches to estimate solubility and permeability in drug discovery and development settings. Adv Drug Deliv Rev. 2001; 46(1-3):3-26.

19. Muegge I. Selection criteria for drug-like compounds. Med Res Rev. 2003; 23(3):302-321.

20. Molinspiration.molinspiration cheminformatics. https:// molinspiration.com/cgi-bin/properties. 2020

21. Karmakar B, Talukdar P, Talapatra SN. An in silico study for two antiinflammatory flavonoids of Nerium oleander on proinflammatory receptors. Research Journal of Life Sciences, Bioinformatics, Pharmaceuticals and Chemical Sciences. 2019;5(1):582-596.

22. Mahesh kumar MVS, Rajagopal SV. Antioxidant, antimicrobial, antiproliferative activities and in silico drug likeness prediction of purified MHH compound, isolated from millingtonia hortensis linn. Int j pharm sci. 2015;7(9):251-257.

23. Madeswaran A, Umamaheswari M, Asokkumar K, Sivashanmugam T, Subhadradevi V, Jagannath P. Docking studies: In silico phosphodiesterase inhibitory activity of commercially available flavonoids. ||| Bangladesh Journal of Pharmacology|||. 2012 May 29;7(1):70-75.

24. Trott 0, Olson A.J. AutoDock Vina: improving the speed and accuracy of docking with a new scoring function, efficient optimization, and multithreading. J. Comput. Chem. 2010;31: 455-461.

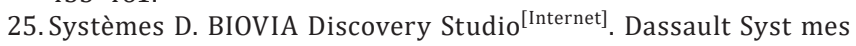
BIOVIA, Discovery Studio Modeling Environment, Release 2017. San Diego: Dassault Syst mes; 2016. Available from: http://accelrys. com/products/collaborative-science/biovia-discovery-studio/

26. Qasaymeh RM, Rotondo D, Oosthuizen CB, Lall N, Seidel V. Predictive Binding Affinity of Plant-Derived Natural Products Towards the Protein Kinase G Enzyme of Mycobacterium tuberculosis (MtPknG). Plants (Basel). 2019; 8(11):477.

27. Meng XY, Zhang HX, Mezei M, Cui M. molecular docking: a powerful approach for structure-based drug discovery. Curr Comput Aided Drug Des. 2011; 7(2):146-57. 